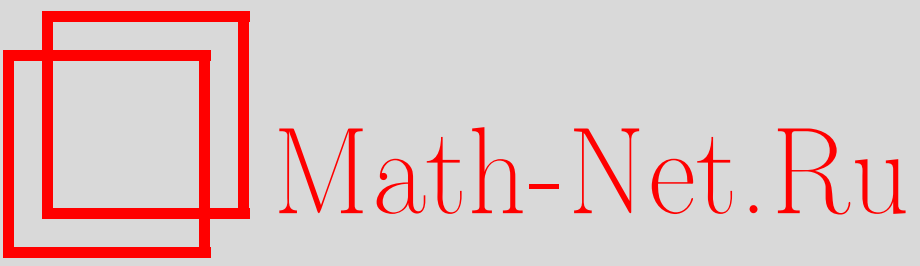

Е. А. Васильева, Эндоморфизмы свободных суперкоммутативных алгебр, УМH, 2000, том 55, выпуск 1, 175-176

DOI: https://doi.org/10.4213/rm263

Использование Общероссийского математического портала Math-Net.Ru подразумевает, что вы прочитали и согласны с пользовательским соглашением

http://www.mathnet.ru/rus/agreement

Параметры загрузки:

IP: 3.80 .253 .173

26 апреля 2023 г., 13:20:51 


\section{ЭНДОМОРфИЗМЫ СВОБОДНЫХ СУПЕРКОММУТАТИВНЫХ АЛГЕБР}

\section{Е. А. ВАСИЛЬЕВА}

Свободные суперкоммутативные ( $\varepsilon$-коммутативные) ассоциативные $K$-алгебры с множеством $X$ свободных порождающих являются в общем случае универсальными обертывающими алгебрами абелевых цветных супералгебр Ли. В недавней работе [1] были введены стандартные базисы таких алгебр, доказан критерий того, чтобы подмножество идеала являлось стандартным базисом этого идеала, получены алгоритмы построения стандартных базисов. В данной заметке техника стандартных базисов в суперкоммутативных алгебрах применяется нами для того, чтобы определить, является ли заданный эндоморфизм суперкоммутативной алгебры сюръективным.

Пусть $K$-поле, $\operatorname{char} K \neq 2,3, G$-абелева полугруппа, $\varepsilon: G \times G \rightarrow K^{*}$-кососимметрическая билинейная форма на $G$ такая, что

$$
\begin{gathered}
\varepsilon(x, y) \varepsilon(y, x)=1, \\
\varepsilon\left(x_{1}+x_{2}, y\right)=\varepsilon\left(x_{1}, y\right) \varepsilon\left(x_{2}, y\right), \\
\varepsilon\left(x, y_{1}+y_{2}\right)=\varepsilon\left(x, y_{1}\right) \varepsilon\left(x, y_{2}\right)
\end{gathered}
$$

для любых $x, x_{1}, x_{2}, y, y_{1}, y_{2} \in G$. Обозначим

$$
G_{-}=\{g \in G \mid \varepsilon(g, g)=-1\}, \quad G_{+}=\{g \in G \mid \varepsilon(g, g)=+1\} .
$$

Пусть $X=\bigcup_{g \in G} X_{g}=\left\{x_{1}, \ldots, x_{n}\right\}-G$-градуированное множество. Определим функцию степени $d: X \stackrel{\longrightarrow}{\longrightarrow}$ соотношением $d(x)=g$ для всех $x \in X_{g}$. Обозначим через $\langle X\rangle$ полугруппу ассоциативных слов из алфавита $X$. Продолжим $d$ с множества $X$ на $\langle X\rangle$, полагая $d(u)=$ $\sum_{i=1}^{n} d\left(x_{i}\right) \in G$ для $u=x_{1} \ldots x_{n} \in\langle X\rangle$.

Пусть $(\langle X\rangle)_{g}=\{u \in\langle X\rangle \mid d(u)=g\},(A\langle X\rangle)_{g}-K$-линейная оболочка элементов из $(\langle X\rangle) g$. Тогда $A\langle X\rangle=\bigoplus_{g \in G}(A\langle X\rangle)_{g}$ - свободная ассоциативная $G$-градуированная алгебра. Пусть $J$ - двусторонний $G$-градуированный идеал в $A\langle X\rangle$, порожденный однородными элементами вида

$$
a b-\varepsilon(d(a), d(b)) b a, \quad a, b \in\langle X\rangle .
$$

Фактор-алгебру $A\langle X\rangle / J$ будем обозначать $K_{\varepsilon}[X]$. Алгебра $K_{\varepsilon}[X]$ является свободной $\varepsilon$-коммутативной ассоциативной $K$-алгеброй с множеством $X$ свободных порождающих. Если $G_{-}=\varnothing$, то $K_{\varepsilon}[X]$ является алгеброй квантовых полиномов. Если $\varepsilon \equiv 1$, то $K_{\varepsilon}[X]$ - обычная полиномиальная алгебра.

Обозначим через $\pi: A\langle X\rangle \rightarrow K_{\varepsilon}[X]$ естественный гомоморфизм. Пусть $X$ - линейно упорядоченное множество. Будем рассматривать следующий порядок на $\langle X\rangle$ : сначала слова упорядочиваются по длине, а затем лексикограффически. Этот порядок естественным образом индуширует порядок на $\pi(\langle X\rangle)$. Дополнительно мы полагаем, что $u>0$ для любого ненулевого $u \in \pi(\langle X\rangle)$.

Пусть $\psi$ - эндоморфизм алгебры $K_{\varepsilon}[X]$, сохраняюший градуировку и удовлетворяюший условию $\psi \mid K=\mathrm{id}_{K}$. Любой такой эндоморфизм имеет вид

$$
h \mapsto h\left(f_{1}, \ldots, f_{n}\right),
$$

где $f_{i}=\psi\left(x_{i}\right), 1 \leqslant i \leqslant n$, и все элементы $f_{i}$ являются однородными степени $d\left(x_{i}\right)$.

Существование $g_{1}, \ldots, g_{n}$ таких, что $g_{i}\left(f_{1}, \ldots, f_{n}\right)=x_{i}$, эквивалентно сюръективности эндоморфизма $\psi$.

Работа выполнена при поддержке Российского фонда фундаментальных исследований (грант № 96-01-00627). 
Пусть $f_{1}, \ldots, f_{m}$ - однородные элементы алгебры $K_{\varepsilon}[X]$. Обозначим через $l p\left(f_{i}\right)$ старший моном полинома $f_{i}$. Введем специальные переменные $t_{1}, \ldots, t_{m}$, каждая из которых соответствует одному из однородных элементов $f_{i}$, и положим $d\left(t_{i}\right)=d\left(f_{i}\right)=d\left(l p\left(f_{i}\right)\right)$. Рассмотрим алгебpy $K_{\varepsilon}[T, X]=K_{\varepsilon}\left[t_{1}, \ldots, t_{m}, x_{1}, \ldots, x_{n}\right]$ и алгебру $K_{\varepsilon}[T]$. Зададим порядок на $K_{\varepsilon}[T, X]$ такой, что

$$
x_{i}>K_{\varepsilon}[T]
$$

для любого $i=1, \ldots, n$. Например, положим $t_{1}<\cdots<t_{m}<x_{1}<\cdots<x_{n}$ и возьмем лексикографический порядок на мономах. Обозначим через $L$ идеал в $K_{\varepsilon}[T, X]$, порожденный множеством

$$
\left\{f_{1}-t_{1}, \ldots, f_{m}-t_{m}\right\},
$$

и пусть $H$ - стандартный базис $L$. Введем следующие обозначения:

$$
\begin{gathered}
H_{T}=H \cap K_{\varepsilon}[T], \quad L p\left(H_{T}\right)=\left\{l p(h) \mid h \in H_{T}\right\}, \\
H_{M}=\left\{h \in H \mid l p(g) \text { не делится ни на один элемент } L p\left(H_{T}\right)\right\} .
\end{gathered}
$$

Легко видеть, что если $H$ - редуцированный стандартный базис $L$, то $H_{M}$ является теоретико-множественным дополнением $H_{T}$.

В недавней работе [2] Д. Шэннона и М. Свидлера построен алгоритм, позволяющий для данных полинома и множества элементов алгебры многочленов определить, допускает ли он представление в виде полинома от этих элементов как новых переменных, и в случае положительного ответа найти это представление в явном виде. В случае суперкоммутативной алгебры многочленов мы воспользуемся следующим резултатом, который является аналогом теоремы, дающей обоснование описанного алгоритма (см. [2]).

Теорема 1. Пусть $h \in K_{\varepsilon}[X]$ u $g \in K_{\varepsilon}[T, X]$ - нормальная форма әлемента $h$ по модулю $H$. Тогда $h \in K_{\varepsilon}\left[f_{1}, \ldots, f_{m}\right]$ тогда и только тогда, когда $g \in K_{\varepsilon}[T]$, при этом $h=g\left(f_{1}, \ldots, f_{m}\right)$.

Доказательство проводится по той же схеме, что и в коммутативном случае.

Теперь мы можем перейти к изложению основной теоремы.

Теорема 2. Следующие условия әквивалентны:

(1) эндоморфизм $\psi$ алгебры $K_{\varepsilon}[X]$ сюрвективен;

(2) множество $H_{M}$ имеет вид $H_{M}=\left\{x_{1}-g_{1}, \ldots, x_{n}-g_{n}\right\}$, где $g_{i} \in K_{\varepsilon}[T]$.

Доказательство импликации $(1) \Rightarrow(2)$ теоремы основано на том, что для суперкоммутативной алгебры $K_{\varepsilon}[X]$ условие $(2)$ эквивалентно условию $K_{\varepsilon}\left[f_{1}, \ldots, f_{n}\right]=K_{\varepsilon}[X]$. Рассуждение опирается на теорему 1 и существенно использует свойства введенного порядка на мономах алгебры $K_{\varepsilon}[T, X]$.

Импликации $(2) \Rightarrow(1)$ доказьвается непосредственным вычислением, на последнем шаге которого мы получаем соотношение, эквивалентное условию (1) теоремы.

Автор выражает искреннюю благодарность своим научным руководителям А. А. Михалеву и А.В. Михалеву за внимание к работе.

\section{СПИСОК ЛИТЕРАТУРЫ}

[1] Mikhalev A. A., Vasilieva E. A. // Proceedings of 2nd International Tainan-Moscow Algebra Workshop. Hong Kong: Springer-Verlag, 1999. P. 108-126. [2] Shannon D., Sweedler M. // J. Symbolic Comput. 1988. V. 6. № 2-3. P. 267-273. 